\title{
Analysis of Coordinated Operation of the Clean Energy System Based on the Multiobjective Optimization Model
}

\author{
Xunyang Wang $\mathbb{D}^{1,2,3}{ }^{1,2 h i c h e n g ~} \mathrm{Ma},{ }^{1}$ Chen Liang, ${ }^{1}$ Shien He, ${ }^{1}$ and Yongqiang Wang ${ }^{3}$ \\ ${ }^{1}$ Postdoctoral Research Station of State Grid Gansu Electric Power Research Institute, No. 249, Wanxin North Road, \\ 730070 Anning District, Lanzhou 730000, Gansu, China \\ ${ }^{2}$ Department of Applied Mathematics, Lanzhou University of Technology, Lanzhou 730050, Gansu, China \\ ${ }^{3}$ College of Electrical and Information Engineering, Lanzhou University of Technology, Lanzhou, Gansu 730000, China
}

Correspondence should be addressed to Xunyang Wang; 12198114@163.com

Received 22 January 2021; Revised 28 March 2021; Accepted 7 April 2021; Published 20 April 2021

Academic Editor: Polinpapilinho Katina

Copyright (c) 2021 Xunyang Wang et al. This is an open access article distributed under the Creative Commons Attribution License, which permits unrestricted use, distribution, and reproduction in any medium, provided the original work is properly cited.

\begin{abstract}
With the increase in the proportion of clean energy connected to the grid, the effective coordination of the operation of various energy power has become a new challenge facing the current power system scheduling. The coordinated operation of the clean energy power generation system can alleviate the contradiction between power generation and output power fluctuations and overcome the bottleneck of new energy development. Considering the natural characteristics of clean energy, this paper aims to make full use of clean energy, reduce system operating costs, increase system power generation, and reduce output fluctuations; we establish a multiobjective optimization model for coordinated scheduling of clean energy power systems. The model seeks to maximize power generation and minimize output fluctuations, power purchase costs, and maintenance costs under the constraints of the grid structure. In this paper, the GA_PSO joint algorithm has an accelerated effect on the target optimization calculation, and then the superiority of the GA_PSO algorithm is verified by the IEEE14 standard system. The standard IEEE39 node test system is used to verify the rationality and feasibility of the model built and provides a reference strategy for the coordinated operation mechanism of the clean energy system. According to the model, in the example in this paper, the maximum value of photovoltaic power prediction is $1290 \mathrm{MW}$, and the minimum value is $210 \mathrm{MW}$; the maximum value of wind power prediction is $780 \mathrm{MW}$, and the minimum value is $28 \mathrm{MW}$; the minimum cost of power purchase and maintenance is $56,950.395$; the maximum generating capacity is $5.045 \mathrm{GW}$; the minimum output fluctuation is $0.120 \mathrm{GW}$.
\end{abstract}

\section{Introduction}

Large-scale wind and photovoltaic grid integration is of great significance to power energy saving and emission reduction, but the uncertainty and limited storability of wind-solarwater energy power generation have led to the complexity of power system scheduling and output fluctuations and power reduction related issues [1], which severely hinder the use of renewable energy and slow down the speed of the reduction of carbon emissions. The coordinated operation of hydropower and intermittent renewable energy can effectively solve these problems. Through modeling and optimizing the coordinated operation of the wind-solar-water system [2], a multisource coordination mechanism was discussed and studied to improve the utilization rate of clean energy and achieve a large-scale energy optimization configuration.

Currently, the research on wind-photoelectric fields in China and abroad is mainly based on economic dispatch $[3,4]$. In recent years, attention has been focused on the theoretical characterization of the stochastic characteristics of wind power. Focusing on water-wind, water-photovoltaic, or water-wind-photovoltaic system, the coordinated operation was modeled and optimized [5]. The goals of the current model usually include power generation, economic benefits, emission reductions, water supply, power generation costs, power reduction, production fluctuations, and flow patterns of hydropower generation. At present, the goal of the model is usually to reflect the effect of power system 
coordinated operation in many aspects, such as power generation, economic benefits, emission reduction, water supply, power generation cost, power reduction, output fluctuation, and flow pattern of hydropower flow $[6,7]$. Since the cost of hydropower generation system is low and almost constant in the short term, most of the optimization objectives of the model are focused on economic dispatch [8]. The main purpose of multienergy coordination is to ensure the stability of the power grid system under the premise of regulating the generation of various energy sources and reducing output fluctuations and to provide a theoretical basis for the construction and development of integrated energy systems. With the rapid development of Chinese wind-solar-water-heat clean energy and the lag of power grid construction, power grid constraints, including the power framework and power transmission capacity, have become the main constraints for coordinated operation.

Most of the earlier studies focused on increasing the profit of power generation and reducing output fluctuations but did not consider the relationship between power generation and output fluctuations [9]. Actually, due to the randomness of wind and solar radiation, generating more power led to increasing output fluctuations. In order to meet the needs of users for various energy sources, in literature [10], the authors established a joint scheduling model of regional integrated energy system (IES for short) with renewable energy and combined cooling heating and power (CCHP for short) and energy storage system, which mainly focused on the energy conversion efficiency and economic benefits of energy conversion equipment and energy storage equipment, but they did not consider the impact of the volatility of different output objects on the whole energy system. In [11], taking the constraints of multiple types of power generation and energy storage into consideration, a model with renewable energy and cooling, heating, and power was established to minimize the overall operating cost, but the randomness and output fluctuation of clean energy were not considered, which increased the difficulty of IES scheduling, and seriously affected the balance between power generation and output fluctuation. The coordinated operation of clean energy followed the Pareto correlation to effectively balance the relationship between multiple targets and the degree of dependence [12], but the study did not take into account different resource changes in different seasons. Moreover, when the transmission capacity of the power grid reached the limit, there was no reduction in power distribution among the various power stations, which was unavoidable for the lag of power grid construction; at the same time, the network architecture of the power system studied was mostly single-layer, which could not reflect the embedding structures of the power grid.

Based on the above literatures, the current research objectives are mainly concentrated in a single dimension. In the current situation of large-scale integrated energy access to the grid, this paper will consider multidimensional research from the energy system to further improve the whole energy system architecture, with multiple objectives of energy system stability, economic benefits, and system power generation as the research object. This paper will mainly study from the following three dimensions: (1) In the case of a variety of energy into the grid, we further discuss the relationship between the energy generation and output fluctuation in order to minimize the output fluctuation. (2) To optimize the economic benefits of the whole system, the main objective is to minimize the power purchase cost and equipment maintenance cost of the main power grid so as to improve the economic benefits of system. (3) This paper explores the detailed coordinated operation mode of the multienergy system, comprehensively considers the typical resource conditions in different seasons, extracts the multilayer power grid structure, and establishes the multiobjective model of the coordinated operation of the integrated energy system. In view of the above problems, considering the advantages of large-scale integrated energy access to the grid, IEEE39 will be adopted to test the rationality and feasibility of the model and algorithm. In this paper, we hold a view to providing data support and application guidance for the coordinated operation of the future energy interconnection system.

The rest of this paper is arranged as follows: in Section 2, the description and establishment of the basic principles of the model are involved; in Section 3, the solution steps of the model are demonstrated in detail; in Section 4, taking the IEEE39 node test system as an example, we comprehensively discuss the research results of the energy system coordination mechanism; finally, we come to the conclusions in this paper.

\section{Multiobjective Model of the Clean Energy System}

The goal of the water-wind-solar clean energy system coordination model is to maximize the generation of clean energy systems and minimize output fluctuations to maximize economic benefits as well $[13,14]$. Constraint types include resource types (water flow, wind speed, and solar radiation), grid types (power balance, transmission capacity, and power reduction allocation), and power plant types (water level, flow, capacity, and energy utilization factor).

2.1. Objective Function. In this section, we study the objective functions that are crucial for optimal control from three aspects, respectively, that is, power generation, power generation cost, and power system volatility.

First of all, we put forward objective function as shown in equation (1) corresponding to mathematical model (2); the goal in the objective function is to maximize the power generation of the clean energy power generation system. In order to overcome the random characteristics of wind-solar energy, we actively coordinate the power generation of the entire system by reducing the discharge water flow and using the water saved in the reservoir as the residual income of the current optimization range. Because the saved water can be used for power generation in the future and is the result of coordination within the current range, it is converted into power generation and included in the total power generation. 


$$
\max \left[\sum_{i=1}^{N}\left(P_{i}^{c o} \times \Delta t\right)+E_{\mathrm{rem}}\right]
$$

satisfying

$$
\left\{\begin{array}{l}
P_{i}^{c o}=P_{i}^{h}+P_{i}^{w}+P_{i}^{p h} \\
E_{\mathrm{rem}}=\Psi_{\mathrm{rem}} \times \rho_{\mathrm{rem}} \times V_{\mathrm{rem}} \times g \times h_{\mathrm{rem}},
\end{array}\right.
$$

where $i$ represents the number of periods: $i=1,2, \ldots, N$, and $N$ is the biggest number of periods; $\Delta t$ represents the time length of a single period; $P_{i}^{c o}$ denotes total output of hydropower, wind power, and photovoltaic power generation in the $i$-th period; $P_{i}^{h}$ denotes hydropower in the $i$-th period, $h$ is short for hydropower; $P_{i}^{w}$ denotes wind power in the $i$-th period, $w$ is short for wind power; $P_{i}^{p h}$ denotes photovoltaic power in the $i$-th period, $p h$ is short for photovoltaic power; $E_{\text {rem }}$ denotes the remaining water volume in the reservoir; $\Psi_{\text {rem }}$ is conversion coefficient; $\rho_{\text {rem }}$ denotes water density; $V_{\text {rem }}$ is the amount of water remaining in the reservoir at the end of the optimization; $g$ denotes the acceleration of gravity; $h_{\text {rem }}$ denotes the average head of remaining water.

Furthermore, in order to realize the operation of the system economically, efficiently, and environmentally friendly, we can follow the policy of giving priority to wind power and full access to the grid in accordance with the requirements of the Renewable Energy Law. Therefore, we set the main power grid purchase cost and maintenance cost as the second optimization goals as follows [15]:

$$
\min \left(C_{e}+C_{p}\right)
$$

satisfying

$$
\left\{\begin{array}{l}
C_{e}=\sum_{i=1}^{T}\left(\frac{F_{r s}^{i}+F_{r b}^{i}}{2} P_{\text {grid }}^{i}+\frac{F_{r b}^{i}-F_{r s}^{i}}{2}\left|P_{\text {grid }}^{i}\right|\right) \\
C_{p}=\sum_{i=1}^{T}\left(\sum_{d=1}^{M} F_{d}\left|P_{d}^{i}\right|\right)
\end{array}\right.
$$

where $C_{e}$ represents the cost of purchasing electricity and $C_{p}$ represents the cost of equipment maintenance; $P_{\text {grid }}^{i}, F_{r s}^{i}$, and $F_{r b}^{i}$ denote the power purchased, the price of electricity purchased, and the price of electricity sold, respectively, during the period; $F_{d}$ denotes the unit maintenance cost of device $d$; $P_{d}^{i}$ represents the output of device $d$ in the $i$ th period.

Finally, in the current energy system, it is not enough to consider only the output fluctuations of clean energy, since this will bring about huge errors and irrationality to the subsequent research. Therefore, we should take the output fluctuations of thermal power into consideration to derive the minimum output fluctuations of the clean energy system. Thus, we further put forward the objective function as follows:

$$
\min (\alpha \times \beta+\Delta P)
$$

$$
\left\{\begin{array}{l}
\alpha=\sqrt{\frac{1}{N} \sum_{i=1}^{N}\left(P_{i}^{c o}-\overline{P_{i}^{c o}}\right)^{2},} \\
\beta=\sum_{i=1}^{N} \theta_{i}, \\
\Delta P=\sum_{p=1}^{N_{p}} \sum_{i=1}^{N}\left|P_{i,(p+1)}^{G}-P_{i, p}^{G}\right|
\end{array}\right.
$$

where $\alpha, \beta$ denotes quantitative fluctuations and contour fluctuations of $P_{i}^{c o}$, respectively, $\overline{P^{c o}}$ is the mean of $P_{i}^{c o}$, the definitions of parameters such as $\theta_{i}$ can be found in [12], $\Delta P$ is the minimum output fluctuation of the thermal power unit in the adjacent period, $P_{i, p}^{G}$ is the output power of the thermal power unit $p$ in the $i-$ th period, and $N_{p}$ represents the total number of thermal power units.

2.2. Constraints. As mentioned before, constraints are divided into three types [16]. Large and complex grids are composed of simple regional grids with hierarchical interconnections, resulting in coordination between water-windsolar energy sources that may occur at different grid levels. Therefore, there are mainly the following aspects when considering the constraints of multilayer power balance.

(i) Power balance constraints:

$$
\Delta P_{m, i}=\left(P_{m, i}^{h}+P_{m, i}^{w}+P_{m, i}^{p h}+P_{m, i}^{G}+P_{m, i}^{o t h}+\Delta P_{m-1, i}\right)-L_{m, i}
$$

where $L_{m, i}$ represents the load of the $m$ - th layer in the $i$ - th period; $\Delta P_{m, i}$ is the power exchange between layer $m$ and layer $m+1$ in the $i-$ th period. While $\Delta P_{m, i}<0$, it indicates downloading power from the $m+1$ th layer; while $\Delta P_{m, i}>0$, it indicates uploading power from the $m+1$ th layer; while $\Delta P_{m, i}=0$, it indicates no power exchange. $P_{m, i}^{h}, P_{m, i}^{w}, P_{m, i}^{p h}+P_{m, i}^{G}, P_{m, i}^{\text {oth }}$ denote the output of electricity from a hydraulic, wind, photovoltaic, thermal power generation, and other power sources at a node of the $m$-th layer in the $i$-th period, respectively.

(ii) System backup constraints:

$$
\begin{aligned}
& \sum_{p=1}^{N_{p}} u_{p, i}\left(P_{\max , p}^{G}-P_{p, i}^{G}\right)+\sum_{q=1}^{N_{q}} u_{q, i}\left(P_{\max , q}^{h}-P_{q, i}^{h}\right) \\
& \quad \geq k_{d} L_{i}+k_{w} \sum_{k=1}^{N_{k}} P_{k, i}^{w} .
\end{aligned}
$$

satisfying 
where $u_{p, i}$ is the on-off state of the thermal power unit $p$ in the $i$ - th period, 1 stands for booting and 0 stands for out of service; $P_{\max , p}^{G}$ represents the maximum output power of thermal power unit $p$; $P_{\max , q}^{h}$ represents the maximum output power of hydropower power unit $q ; N_{p}, N_{k}$ denote the total numbers of hydropower units and wind power units, respectively. $k_{d}, k_{w}$ represent load fluctuation coefficient and wind power fluctuation coefficient, respectively, and we value them as $k_{d}=10 \%$ and $k_{w}=15 \%$. The reserve capacity is required to meet the random fluctuations of wind power and load.

(iii) The upper and lower limits of thermal power output:

$$
P_{\min , p}^{G} \leq P_{i, p}^{G} \leq P_{\max , p}^{G}
$$

where $P_{\min , p}^{G}$ and $P_{\max , p}^{G}$ represent the minimum and maximum output power of thermal power unit $p$, respectively.

(iv) Hydropower generation capacity and conversion efficiency constraints:

$$
\left\{\begin{array}{l}
P_{\min , q}^{h} \leq P_{i, q}^{h} \leq P_{\max , q}^{h}, \\
P_{i, q}^{h}=A \times \lambda_{h} \times \times Q_{i, q} h_{i, q} .
\end{array}\right.
$$

where $P_{\min , q}^{h}$ and $P_{\max , q}^{h}$ represent the minimum and maximum output power of the hydropower unit $q$, respectively; $A$ represents hydroelectric conversion constant; $Q_{i, q}$ denotes the water consumption of reservoir $q$ in the $i$ - th period; $h_{i, q}$ represents the head height of the reservoir $q$ in the $i$ - th period.

(v) Wind power capacity constraints:

$$
\left\{\begin{array}{l}
P_{\min , k}^{w} \leq P_{i, k}^{w} \leq P_{\max , k}^{w} \\
P_{i, k}^{w}= \begin{cases}0, & v_{i}<v_{\text {in }} \text { or } v_{i}>v_{\text {cut }} \\
g\left(v_{i}\right), & v_{\text {in }}<v_{i}<v_{\text {out }} .\end{cases}
\end{array}\right.
$$

where $P_{\min , k}^{w}$ and $P_{\max , k}^{w}$ denote the upper and lower limits of the wind turbine output $k$, respectively; $v_{\text {in }}$ and $v_{\text {cut }}$ denote the cut-in and cut-out wind speeds of wind turbine generator, respectively; $g\left(v_{i}\right)$ describes the relationship between wind energy output and wind speed.

(vi) Photovoltaic power generation capacity constraints:

$$
\left\{\begin{array}{l}
P_{\min }^{p h} \leq P_{i}^{p h} \leq P_{\max }^{p h}, \\
P_{i}^{p h}=\eta_{1} \times \eta_{2} \times \eta_{3} \times R_{i} .
\end{array}\right.
$$

where $P_{\min }^{p h}$ and $P_{\max }^{p h}$ are the upper and lower limits for photovoltaic output, respectively; $\eta_{i}(i=1,2,3)$ represent photovoltaic array coefficient, inverter coefficient, and connection power coefficient, respectively; $R_{i}$ denotes solar radiation intensity.

2.3. Research Objectives. In order to discuss the mechanism and superiority of coordinated operation of multitarget clean energy power generation system, the analysis based on the above model was studied. In this model, the goal is to maximize the power generation and minimize output fluctuations through the coordinated operation of clean energy power generation systems, while minimizing the cost of power purchase and maintenance. The objective functions are as follows:

$$
\begin{aligned}
& \max \left[\sum_{i=1}^{N}\left(P_{i}^{c o} \times \Delta t\right)+E_{\mathrm{rem}}\right], \\
& \min \left(C_{e}+C_{p}\right), \\
& \min (\alpha \times \beta+\Delta P) .
\end{aligned}
$$

\section{Model Solving Algorithm}

The clean energy power generation system is a large-scale system with multiobjective, nonlinear, and strong coupling characteristics. The coordinated operation of the system includes decoupling the network architecture from the lower layer to the upper layer and independent configuration according to the characteristics of the power station, which leads to the problem of multiobjective collaborative optimization which is difficult to converge. Using the elite strategy of simultaneous cloning and evolution and the idea of similar mating [17], the genetic algorithm (GA) and particle swarm optimization (PSO) algorithm are combined to further improve its convergence. Due to the low complexity, fast convergence, and uniform distribution of the Pareto boundary, this paper applies the GA_PSO joint algorithm to solve multiobjective optimization problems. In this study, the MATLAB multiobjective optimization toolbox was used to determine the best coordinated operation of multiobjective optimization.

GA_PSO joint algorithm implementation steps are as follows:

Step 1 (Initialization). For an $n$-dimensional problem, a group with a size of $4 n$ is generated within the feasible domain. In this paper, the clean energy output ratio is used as the initial group. When the grid automatically stratified number $m<4 n$, some points are selected from the random samples as the initial point, and the $4 n$ individuals constitute the evolutionary operation.

Step 2 (Sorting). Calculate the fitness values of $4 n$ individuals and rank them from good to bad.

Step 3 (GA Method). The $2 n$ individuals above are crossmutated with the real-coded GA operator to generate $2 n$ new individuals. 
Step 4. Calculate the fitness value of the new $2 n$ chromosomes generated by GA and replace the worst individual with the elite parent individual; that is, the fitness value is inferior to that of the parent elite individual, and place it in the corresponding position.

Step 5 . The above $2 n$ chromosomes are sorted according to the fitness value and paired with each other. Each team forms a neighborhood and determines the optimal individual.

Step 6 (PSO Method). The best fitness individual in GA algorithm is regarded as the global extremum of the PSO algorithm, and the better fitness value in each neighborhood is regarded as the individual extremum of the neighborhood. Compared with the previous neighborhood optimal particle, the optimal particle is updated in real time.

Step 7. Determine whether the convergence conditions are met. If the convergence condition is satisfied or the number of iterations is reached, the algorithm ends; otherwise, return to step 2 and repeat the above process until the convergence condition is satisfied.

The logic diagram of the GA_PSO joint algorithm is shown in Figure 1.

\section{Example Analysis}

We adopt the IEEE14 node test system appearing in [18] as an example and consider the lowest purchase cost and maintenance cost as the goal. Suppose that the population size is 14 , the maximum number of iterations is 100 , and the calculation time is 25. GA, PSO, and GA_PSO algorithms are used, respectively, for optimization and comparison.

It can be seen from Figure 2 that the GA algorithm has much better robustness, faster convergence, and more reasonable calculation results; PSO calculation functions often have premature phenomena, and the system convergence is rather weak; GA_PSO optimization algorithm has the best iterative convergence, and the system tends to be more stable, so the GA_PSO optimization algorithm has an accelerated effect on the optimization calculation of the function. From the convergence of the above three algorithms, it can be seen that when the lowest power purchase cost and maintenance cost are selected as the objective functions, the proposed GA_PSOjoint algorithm is the most feasible to optimize this model.

\section{Applications on Models and Algorithms}

We adopt IEEE10 machine 39-node standard system model [19] and standard data of the system and use part of the existing data [20] to solve the objective functions proposed in this paper.

Figure 3 shows the predicted power value and the load value in each cycle of the wind farm.

The predicted maximum value of wind power is $780 \mathrm{MW}$, accounting for $78 \%$ of the total installed capacity of the wind farm; the minimum value is $28 \mathrm{MW}$, accounting for $2.4 \%$ of the total installed capacity of the wind farm. It can be

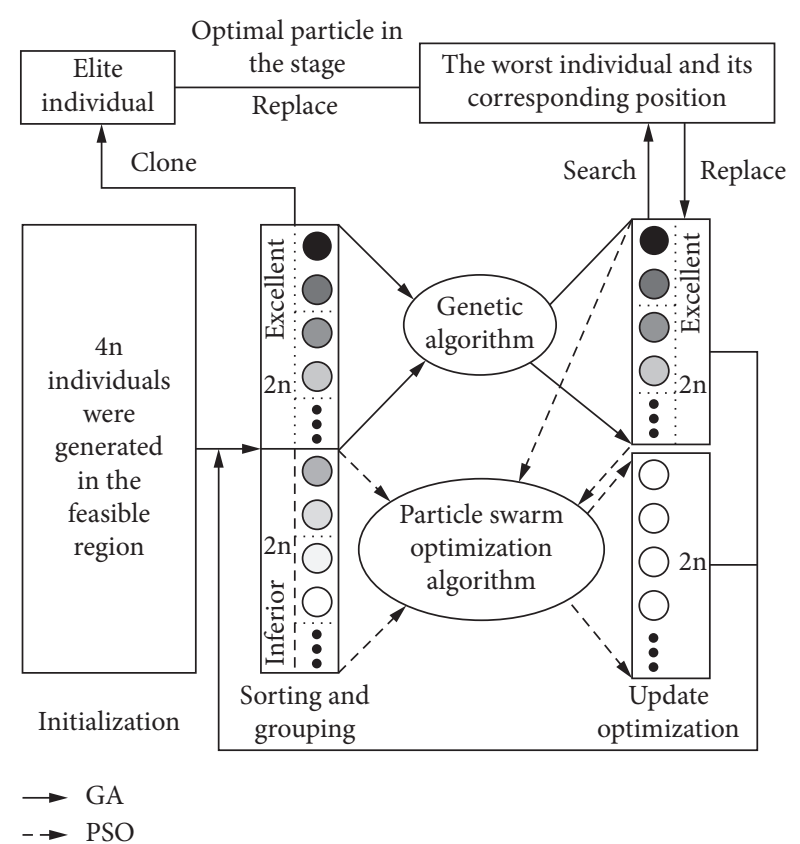

FIgURE 1: The implementation logic diagram of GA_PSO joint algorithm.

seen that the peak-valley difference of the random fluctuation of wind power output is very large, and it can also be seen from Figure 1 that wind power has a significant inverse peak-shaving characteristic relative to the load.

Through the statistics of photovoltaic output, it can be seen from Figure 4 that the photovoltaic power distribution is close to a normal distribution, and, at the same time, the photovoltaic output has strong trend characteristics and obvious random fluctuations. The above analysis provides basic data and reliable theoretical basis. The predicted maximum value of photovoltaic power is $1290 \mathrm{MW}$, and the minimum value is $210 \mathrm{MW}$.

By comparing the data in Table 1, it can be seen that the three optimization algorithms have obtained better results, which fully shows that the GA_PSO joint algorithm increases the particle diversity and effectively improves the defect that the PSO algorithm is easy to fall into the local optimum. Among them, the final optimization result of power purchase cost and maintenance cost based on GA_PSO joint algorithm is $56,950.395$ dollars.

As seen from Figure 5, in view of the surplus water resources in the flood season, the dispatch center considers that, from the safety point of view, the hydropower plant must be full, so the hydropower in the flood season has no regulation capacity. Clean photovoltaic and wind power are given priority, so the optimized variable wind-light output has the smallest fluctuations and ensures the maximum power generation. The output of photovoltaics fluctuates significantly, with a large peak-to-valley difference of 1469.35 MW, which accounts for about $61 \%$ of the maximum output. This shows that, in the case of photovoltaics that do not have the ability to regulate, wind power must undertake the dual task of making up for the random fluctuations of photovoltaics and the difference between 

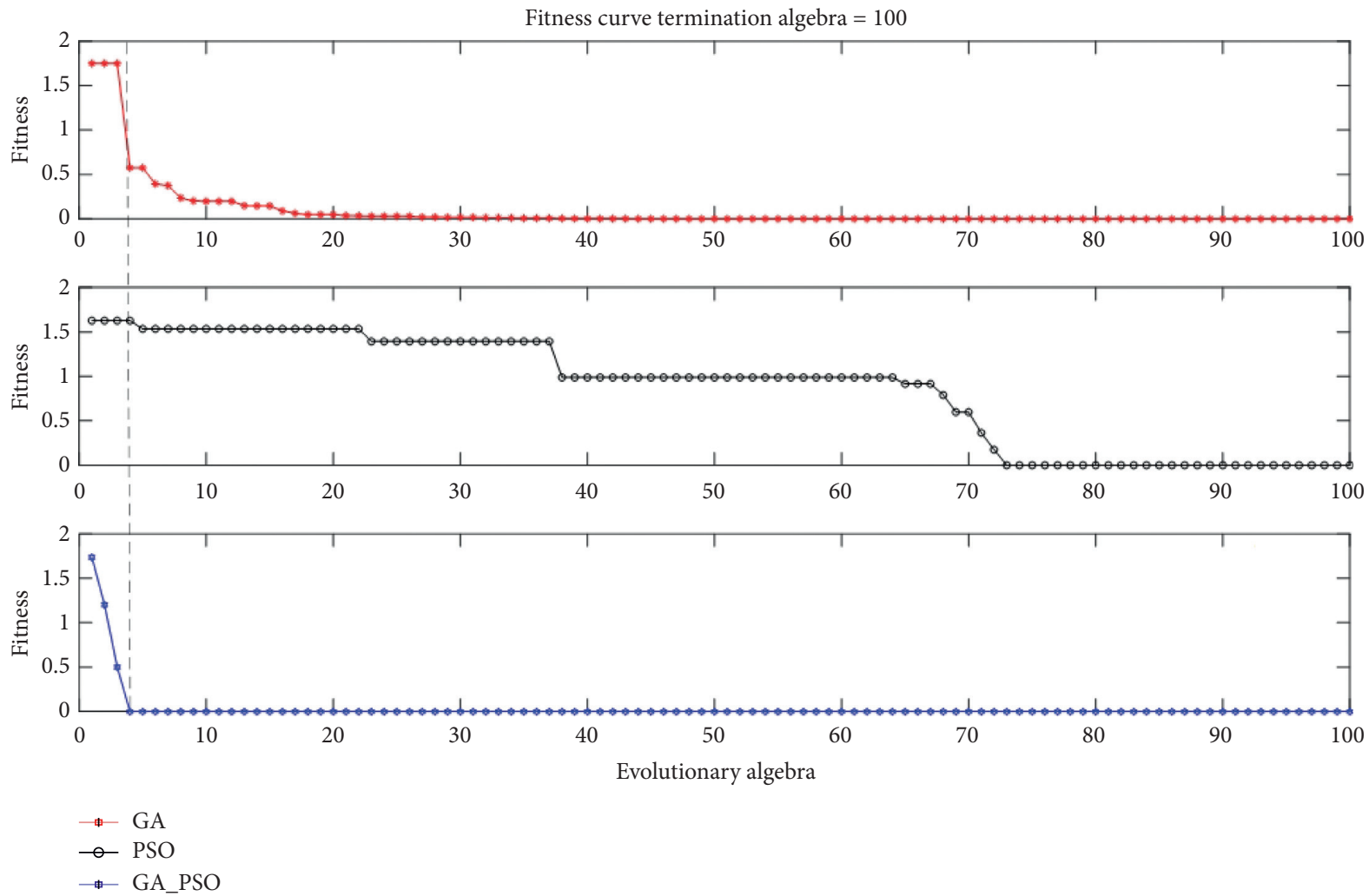

FIgURE 2: Comparison of fitness curves of three algorithms.

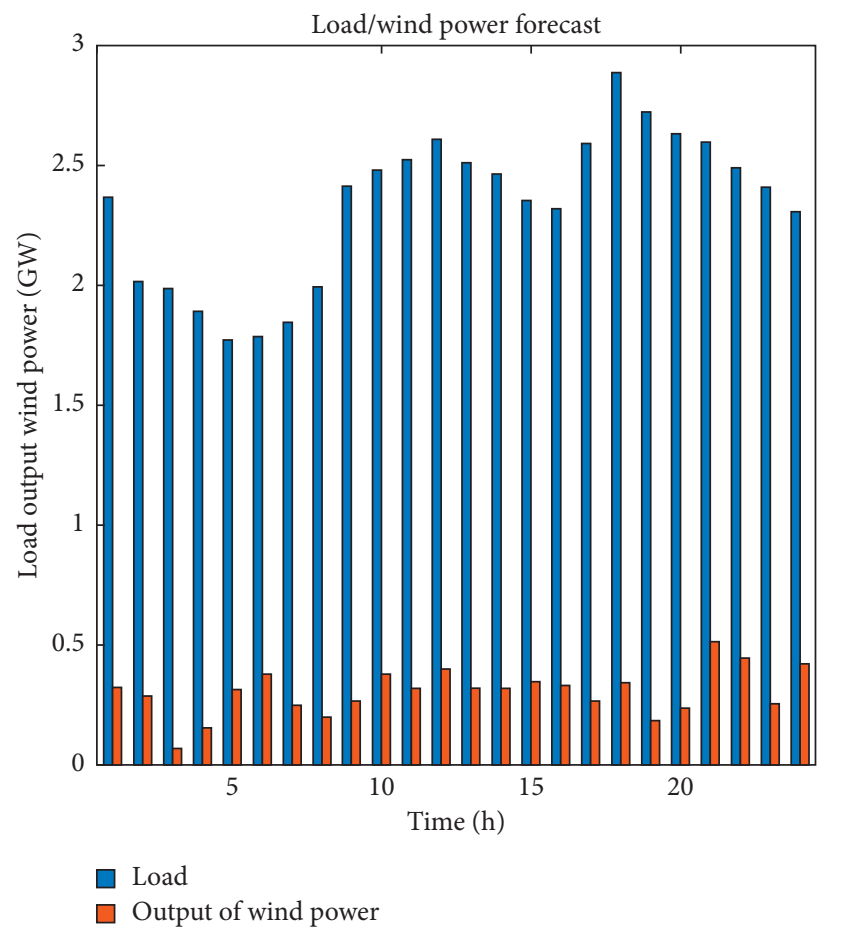

FIGURE 3: Load and wind power forecast.

peaks and valleys in load, which leads to frequent adjustments of wind power output.

In Table 2, the symbol "COV" means "cost optimization value"; the symbol "OVPG" means "optimal value of power generation"; the symbol "OVOF" means "optimal value of output fluctuation."

For the multiobjective optimization of the clean energy system, the relationship between the multiobjective 


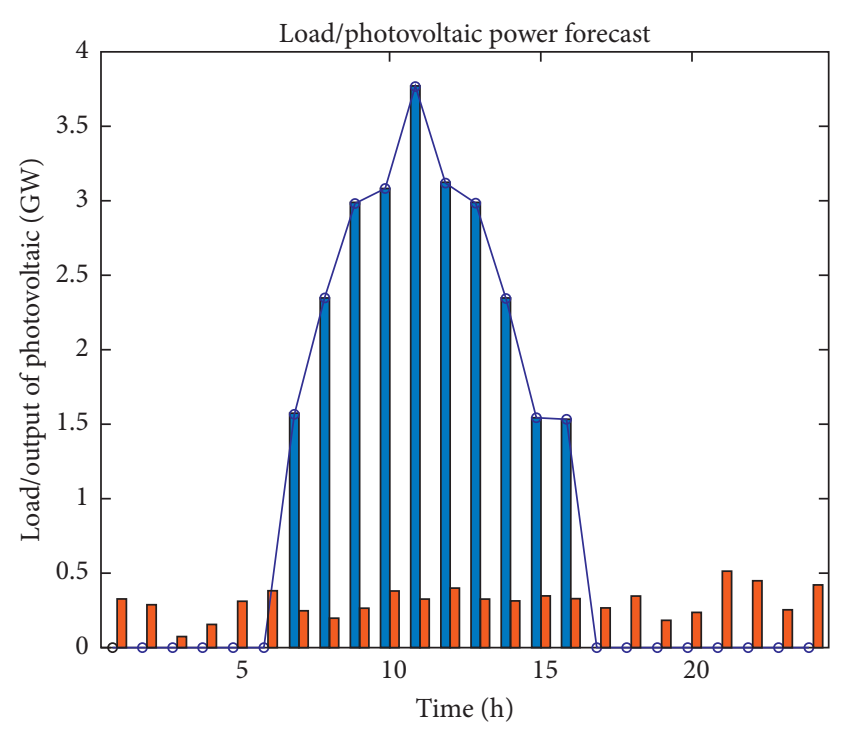

$\square$ Output of photovoltalic

$\square$ Load

FIGURE 4: Load and photovoltaic power prediction.

TABLE 1: Optimization results of power purchase cost and maintenance cost.

\begin{tabular}{lccc}
\hline Algorithm & Maximum value & Minimum value & Average value \\
\hline GA & $58,743.28$ & $57,166.45$ & $57,954.865$ \\
PSO & $58,443.35$ & $56,962.09$ & $57,702.720$ \\
GA_PSO & $57,846.67$ & $56,054.12$ & $56,950.395$ \\
\hline
\end{tabular}

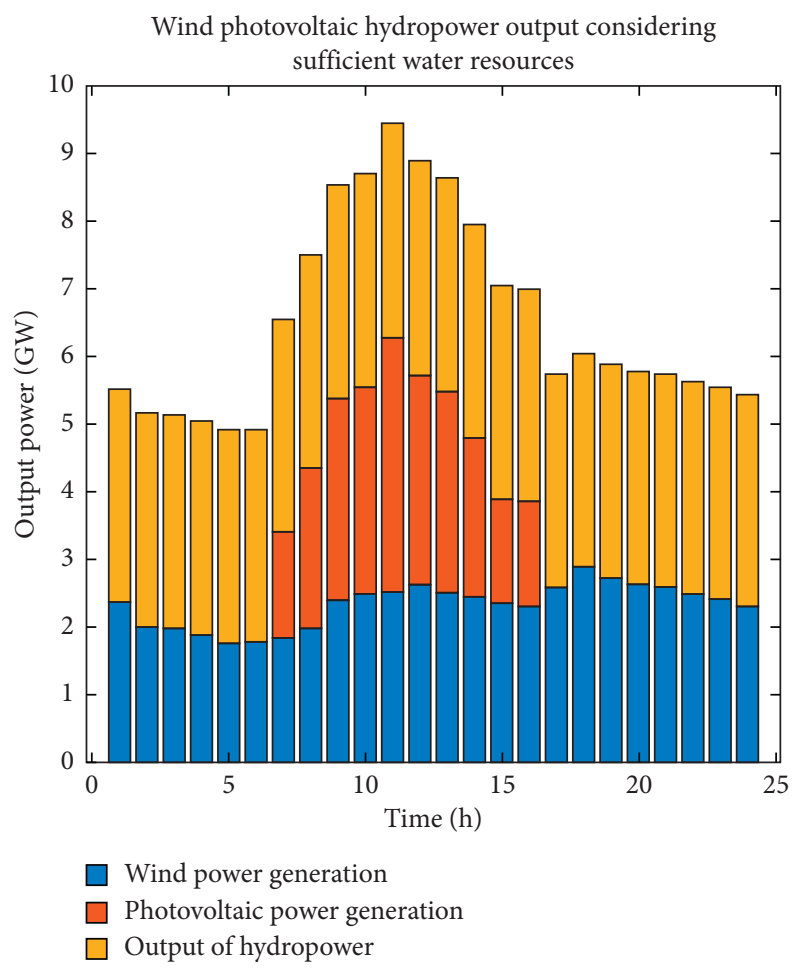

FIgURe 5: Clean energy output.
TABLE 2: Comparison of multiobjective optimization results.

\begin{tabular}{lccc}
\hline Algorithm & COV/\$ & OVPG/GW & OVOF/GW \\
\hline GA & $57,954.865$ & 4.895 & 0.142 \\
PSO & $57,702.720$ & 4.876 & 0.219 \\
GA_PSO & $56,950.395$ & 5.045 & 0.120 \\
\hline
\end{tabular}

optimizations can be obtained through the literature [21] to satisfy the Pareto relationship. Therefore, this paper uses the GA_PSO joint algorithm to optimize power purchase costs and maintenance costs, power generation, and output fluctuations for multiobjective optimization. From Table 2, we can see that the minimum power purchase cost and maintenance cost is 56,950.395; the maximum power generation is $5.045 \mathrm{GW}$; the output fluctuation is minimized to $0.120 \mathrm{GW}$.

\section{Conclusions}

In view of the limitations of the traditional power system clean energy joint scheduling in large-scale clean energy centralized access system and the contradiction between multienergy power generation and output fluctuations, in this paper, we establish a clean energy power generation system model in order to study the coordinated operation mechanism of different energy. Our objectives are to make full use of clean energy, reduce system operating costs, and make use of mutual compensation between different clean energy sources to to avoid output fluctuations and power reduction. The simulation results of the example show the following:

(1) The GA algorithm uses selection, crossover, and mutation operator operations for function optimization and directly uses the target function of the model as search information in a probabilistic manner, thus enhancing the global optimization ability of the PSO optimization algorithm, speeding up the evolution of the algorithm, and improving the convergence accuracy.

(2) The model built in this paper can provide some data supporting for the scheduling strategy of the clean energy system and a strong guarantee for the full coordination of clean energy power generation systems, which can further improve the economic benefits of the power grid and ease the pressure of peak and frequency regulation of the power grid and ensure the maximum utilization of the energy system as well.

(3) Adopting the coordinated operation mechanism of the clean energy power generation system can improve the grid's ability to accept clean energy under the premise of ensuring the operation efficiency and stability of the power grid system and can provide reference strategies for the coordinated operation of other power sources. Additionally, coordinated operation mechanism of new energy power generation also can provide data support and application guidance for the construction and coordinated operation of the energy Internet. 


\section{Data Availability}

Part of the data is acquired from other published papers (cited), and some data are estimated in a reasonable range.

\section{Conflicts of Interest}

The authors declare that they have no conflicts of interest.

\section{Acknowledgments}

This research was supported by the National Natural Science Foundation of China (NSFC) under Grant no. 11661050.

\section{References}

[1] S. Jianjian, Z. Xiufei, C. Rui et al., "Short term practical dispatching method for large-scale hydropower stations in hydropower enrichment power grid," Journal of Chinese Electrical Engineering, vol. 39, no. 10, pp. 2816-2828, 2019.

[2] F. Xiaoyun, L. Lei, Y. Gang et al., "Multi-objective shape optimization of autonomous underwater glider based on fast elitist non-dominated sorting genetic algorithm," Ocean Engineering, vol. 157, 2018.

[3] L. Thomas, A. Acker, H. Robitaille et al., "Integration of wind and hydropower systems: results of IEA wind task 24," Wind Engineering, vol. 36, no. 1, 2012.

[4] W. Kui, Z. Buhan, Z. Yang et al., "Real time scheduling of power system with wind farm based on chaos quantum particle swarm optimization algorithm," Power System Automation, vol. 35, no. 22, pp. 141-146, 2011.

[5] D. Nicola, K. Magnus, and F. Julian, "Smoothing of offshore wind power variations with Norwegian pumped hydro: case study," Energy Procedia, vol. 87, 2016.

[6] P. Sergio, F. Paula, and A. I. F. Vaz, "A simplified optimization model to short-term electricity planning," Energy, vol. 93, 2015.

[7] C. Zheng, Y. Jiajia, J. Xiaoming et al., "Pricing mechanism of renewable energy generation and bidding strategy of participating in electricity market," Journal of North China Electric Power University (NATURAL SCIENCE EDITION), vol. 41, no. 2, pp. 89-98, 2014.

[8] C. Liang, G. Xueping, and J. Jinghua, "Multi objective optimization of extended black start scheme considering subsequent recovery," Journal of Power System and Automation, vol. 26, no. 2, pp. 21-26, 2014.

[9] C. Gonggui and C. Jinfu, "Modeling and algorithm for environmental economic dynamic dispatch of power system with wind farms," Acta Electrical Engineering Sinica, vol. 33, no. 10, pp. 27-35, 2013.

[10] L. Dachen, M. Hengrui, W. Bo et al., "Operation optimization of regional integrated energy system including CCHP and energy storage," Power System Automation, vol. 42, no. 4, pp. 113-120, 2018.

[11] G. Lin, C. Yuwei, L. Yuquan et al., "Collaborative optimization and case analysis of multi energy flow of microgrid with renewable energy," Power Automation Equipment, vol. 37, no. 6, pp. 275-281, 2017.

[12] R. Azizipanah-Abarghooee, N. Taher, R. Alireza et al., "Probabilistic multiobjective wind-thermal economic emission dispatch based on point estimated method," Energy, vol. 37 , no. $1,2011$.
[13] O. A. Jaramillo, M. A. Borja, and J. M. Huacuz, "Using hydropower to complement wind energy: a hybrid system to provide firm power," Renewable Energy, vol. 29, no. 11, 2004.

[14] Z. Yuman, L. Xuezhi, Y. Zheng et al., "Study on decomposition coordination and optimal operation of photovoltaic energy storage cogeneration integrated energy system," Acta Electrotechnics, vol. 1-15, 2020.

[15] Y. Shuo, Z. Peng, Y. Meng et al., "Multi time scale optimal dispatch of integrated energy system considering demand side response," Journal of Power System and Automation, vol. 32, no. 11, pp. 39-46, 2020.

[16] Z. Dan, S. Ke, Z. Quanming et al., "Recent robust optimal scheduling of distribution networks with multiple integrated energy cogeneration microgrids," Acta Electrical Engineering Sinica, vol. 40, no. 14, pp. 4473-4485, 2020.

[17] Z. Ganqing and G. Xiansheng, "Structural collaborative optimization based on hybrid GA_PSOalgorithm in case of variable correlation," Journal of Mechanical Engineering, vol. 48, no. 15, pp. 113-125, 2012.

[18] Retrieved January 8, 2021, from https://www.mathworks. com/matlabcentral/fileexchange/46067-ieee-14-bus-system.

[19] Retrieved January 8, 2021, from https://download.csdn.net/ download/lp19891003/6807051.

[20] Y. Faqi, L. Yaowang, W. Min et al., "Multi time scale power reserve joint optimal dispatch of power system with caes and multiple types of flexible loads," Power Automation Equipment, vol. 39, no. 12, pp. 73-81, 2019.

[21] S. Xin, C. Jinge, Z. Xiaocheng et al., "Multi-objective optimization of wind turbine blades using lifting surface method," Energy, vol. 90, pp. 1111-1121, 2015. 